\title{
Laboratory assessment of fitness to fly in patients with lung disease: a practical approach
}

\author{
A.G. Robson, T.K. Hartung, J.A. Innes
}

\begin{abstract}
Laboratory assessment of fitness to fly in patients with lung disease: a practical approach. A.G. Robson, T.K. Hartung, J.A. Innes. (C)ERS Journals Ltd 2000.

ABSTRACT: To identify patients with respiratory disease, who may be at risk of developing respiratory distress during commercial air travel, a hypoxia inhalation test (HIT) can be performed. This paper reports our experience of using such a test combined with an interpretation algorithm in a routine respiratory function laboratory.

Twenty-eight patients were studied. Baseline oxygen saturation $\left(S_{\mathrm{a}}, \mathrm{O}_{2}\right)$ was measured using a pulse oximeter. If $\mathrm{Sa}_{\mathrm{a}} \mathrm{O}_{2}$ was $<90 \%$ no HIT was performed and the patient was assessed as unfit for air travel. If baseline $S \mathrm{a}, \mathrm{O}_{2}$ was $\geq 90 \%$ an HIT was performed by the patient breathing through a $35 \%$ Venturi mask supplied with $100 \%$ nitrogen which reduced inspiratory oxygen fraction to $15.1 \pm 0.2 \%$. Results were interpreted using a locally derived algorithm, and validation was attempted using a questionnaire to investigate subsequent symptoms during travel.

All patients tolerated the assessment well. Twenty-two patients were assessed as "fit to fly" with a further two patients "fit to fly with supplemental $\mathrm{O}_{2}$ ". Four patients were considered unfit to fly. Hypoxic response could not be predicted from either forced expiratory volume in one second, or pretest saturation.

Validation of such protocols is difficult, but the hypoxia inhalation test may be a useful tool for predicting hypoxia during air travel in patients with chronic respiratory disease.

Eur Respir J 2000; 16: 214-219.
\end{abstract}

Commercial aviation is increasing rapidly with passenger transit through UK airports doubling since 1987 [1]. It follows that increasing numbers of patients with lung disease are considering air travel. At cruising altitudes $(9,150-12,200 \mathrm{~m})$ most commercial aircraft are unable to maintain the internal cabin pressure at sea level $(\sim 100$ $\mathrm{kPa}$, partial pressure of oxygen $\left.\left(\mathrm{PO}_{2}\right) 21 \mathrm{kPa}\right)$ but maintain a pressure which is equivalent to an altitude of 2,438 metres $(8,000$ feet) above sea level [2]. At this pressure the $\mathrm{PO}_{2}$ is $\sim 14.4 \mathrm{kPa}$ which is the equivalent of an inspired fraction of oxygen $\left(F \mathrm{I}, \mathrm{O}_{2}\right)$ of $0.151(15.1 \%)$ at sea level.

Although most passengers can tolerate this reduction in $\mathrm{PO}_{2}$ without experiencing any respiratory distress, passengers with chronic respiratory disease may develop an exacerbation of their symptoms. In extreme cases this may lead to the declaration of a medical emergency and the diversion of the plane to the nearest airport.

To identify persons who are at risk of developing respiratory distress during air travel a number of studies have been performed of the response to hypoxia in patients with chronic respiratory conditions. This is done by either exposing subjects to a low $F \mathrm{I}, \mathrm{O}_{2}$ [3-5] or by using hypobaric chambers [6-8]. Due to the relative inaccessibility of hypobaric chambers the hypoxia inhalation test (HIT) of

For editorial comments see page 197.
Respiratory Function Laboratory, Western General Hospital, Edinburgh, Scotland, UK.

Correspondence: J.A. Innes, Respiratory Medicine Unit, Western General Hospital, Crewe Road South,

Edinburgh

EH4 2XU

UK Fax: 441313433989

Keywords: Air travel hypoxia oxygen therapy

Received: March 311999

Accepted after revision February 52000 the type first described by Gong et al. [4] is a more practical test for use in a routine clinical laboratory.

This paper reports the authors' experience of using an HIT in a clinical situation on a range of patients with chronic respiratory conditions, who were contemplating air travel and had asked the opinion of their physician regarding their fitness to fly.

\section{Methods}

Over an 18-month period, 28 patients (13 male, 15 female, age range 33-89 yrs (see table 1 for patient details)) were referred to the laboratory for assessment of their fitness to fly. Most patients (20, (71\% of population)) had a diagnosis of chronic airflow obstruction, four patients $(14 \%)$ had pulmonary fibrosis, one patient had systemic lupus erythematosis (SLE), one had bronchiolitis, one had bronchiectasis and one patient had mesothelioma. All patients were referred by consultant physicians, primarily at the request of the patients themselves who had sought medical advice before travelling.

Spirometry was performed on all patients before assessment using a wedge bellows spirometer (Vitalograph Model R; Vitalograph, UK). Forced expiratory volume in one second (FEV1) and vital capacity (VC) were measured and compared with the predicted values quoted by CRAPO et al. [9] (males) and HALl et al. [10] (females). All blood gas analyses were performed on a Chiron 248 blood gas 
Table 1. - Patient data, including diagnosis and the result of the assessment

\begin{tabular}{|c|c|c|c|c|c|c|c|c|c|c|}
\hline $\begin{array}{l}\text { Patient } \\
\text { number }\end{array}$ & Age & Sex & Diagnosis & Smoking status & FEV1 & $\mathrm{VC}$ & $\begin{array}{c}\mathrm{FEV} 1 / \mathrm{VC} \\
\text { ratio }\end{array}$ & $\begin{array}{c}\text { FEV1 } \\
\text { (\% pred)* }\end{array}$ & $\begin{array}{c}\text { VC } \\
(\% \text { pred })^{*}\end{array}$ & $\begin{array}{l}\text { Assessment } \\
\text { group }\end{array}$ \\
\hline 1 & 89 & $\mathrm{~F}$ & Bronchiectasis & Nonsmoker & 0.70 & 1.60 & 44 & 56 & 80 & A \\
\hline 2 & 33 & $\mathrm{~F}$ & Bronchiolitis & Nonsmoker & 0.95 & 2.30 & 41 & 34 & 65 & A \\
\hline 3 & 54 & $\mathrm{M}$ & COPD & Exsmoker & 0.50 & 3.75 & 13 & 13 & 80 & A \\
\hline 4 & 52 & M & COPD & Exsmoker & 0.70 & 2.95 & 24 & 18 & 60 & A \\
\hline 5 & 70 & M & COPD & Exsmoker & 0.50 & 1.25 & 40 & 22 & 45 & $\mathrm{C}$ \\
\hline 6 & 64 & $\mathrm{~F}$ & COPD & Exsmoker & 0.50 & 1.35 & 37 & 26 & 51 & A \\
\hline 7 & 65 & M & COPD & Exsmoker & 0.90 & 2.35 & 38 & 27 & 55 & B \\
\hline 8 & 69 & $\mathrm{M}$ & COPD & Smoker & 0.90 & 2.40 & 38 & 28 & 59 & A \\
\hline 9 & 69 & M & COPD & Smoker & 1.00 & 3.70 & 27 & 29 & 84 & A \\
\hline 10 & 57 & $\mathrm{~F}$ & COPD & Smoker & 0.65 & 1.70 & 38 & 31 & 61 & $\mathrm{C}$ \\
\hline 11 & 64 & $\mathrm{~F}$ & COPD & Smoker & 0.60 & 2.15 & 28 & 31 & 82 & A \\
\hline 12 & 70 & M & COPD & Smoker & 0.95 & 2.65 & 36 & 31 & 67 & A \\
\hline 13 & 70 & $\mathrm{M}$ & COPD & Exsmoker & 1.10 & 2.00 & 55 & 36 & 51 & A \\
\hline 14 & 72 & M & COPD & Exsmoker & 1.25 & 3.40 & 37 & 40 & 84 & A \\
\hline 15 & 68 & $\mathrm{~F}$ & COPD & Smoker & 0.85 & 1.35 & 63 & 40 & 46 & $\mathrm{C}$ \\
\hline 16 & 65 & $\mathrm{~F}$ & COPD & Smoker & 0.90 & 2.20 & 41 & 42 & 75 & A \\
\hline 17 & 75 & $\mathrm{~F}$ & COPD & Exsmoker & 0.70 & 1.85 & 38 & 45 & 82 & $\mathrm{C}$ \\
\hline 18 & 73 & M & COPD & Exsmoker & 1.85 & 4.10 & 45 & 58 & 98 & B \\
\hline 19 & 77 & $\mathrm{~F}$ & COPD & Exsmoker & 1.00 & 1.85 & 54 & 60 & 76 & A \\
\hline 20 & 71 & $\mathrm{~F}$ & COPD & Exsmoker & 1.20 & 2.45 & 49 & 63 & 91 & A \\
\hline 21 & 56 & $\mathrm{~F}$ & COPD & Exsmoker & 1.45 & 2.70 & 54 & 65 & 92 & A \\
\hline 22 & 82 & $\mathrm{M}$ & COPD & Exsmoker & 1.70 & 3.45 & 49 & 67 & 104 & A \\
\hline 23 & 68 & M & Mesothelioma & Exsmoker & 1.50 & 1.80 & 83 & 45 & 42 & A \\
\hline 24 & 53 & $\mathrm{~F}$ & Pulmonary fibrosis & Nonsmoker & 1.50 & 2.20 & 68 & 66 & 74 & A \\
\hline 25 & 57 & $\mathrm{~F}$ & Pulmonary fibrosis & Exsmoker & 1.40 & 1.65 & 85 & 69 & 61 & A \\
\hline 26 & 73 & M & Pulmonary fibrosis & Nonsmoker & 2.05 & 2.55 & 80 & 73 & 71 & A \\
\hline 27 & 76 & $\mathrm{~F}$ & Pulmonary fibrosis & Nonsmoker & 1.50 & 1.70 & 88 & 117 & 91 & A \\
\hline 28 & 43 & $\mathrm{~F}$ & SLE & Exsmoker & 1.05 & 1.35 & 78 & 40 & 44 & A \\
\hline
\end{tabular}

A: fit to fly; B: fit to fly with supplemental $\mathrm{O}_{2}$; C: unfit to fly; COPD: chronic obstructive pulmonary disease; SLE: systemic lupus erythrematosis: M: male; F: female; FEV1: forced expiratory volume in one second; VC: vital capacity; *: values based on predicted values from $[9,10]$.

analyser (Chiron Diagnostics, Halstead, UK) which was calibrated daily.

Hypoxic challenge was performed using a modification of the method of VOHRA and KLOCKE [11] in which a Venturi mask designed for controlled oxygen delivery is supplied instead with nitrogen (see below). The principle of using hypoxic challenge to assess patients with lung disease is well established and has passed ethics review [4]. The precise method used in the present study to perform hypoxic challenge has also passed ethics review [11].

Fitness to fly was judged from the results of the hypoxic challenge using a locally derived algorithm. This algorithm was constructed using clinical experience with the aim of achieving reliable prediction of in-flight hypoxia whilst minimizing the need for arterial blood gas sampling.

\section{Validation of hypoxic challenge method}

In order to assess the accuracy and reproducibility of the technique described by VoHra and KLOCKe [4], FI, $\mathrm{O}_{2}$ at the mouth was measured in healthy volunteers using a zirconium oxide oxygen sensor (Benchmark, PK Morgan, Rainham, UK) whilst the volunteers wore a full face Venturi mask (Hospital and Medical Supplies, Glasgow, UK) supplied with $100 \% \mathrm{~N}_{2}$. Pilot testing showed that a mask designed to deliver $35 \%$ oxygen produced an acceptable reduction in $\mathrm{FI}, \mathrm{O}_{2}$ to around $15 \%$ when supplied with $8 \mathrm{~L} \cdot \mathrm{min}^{-1}$ of $100 \% \mathrm{~N}_{2}$. The stability of the $F \mathrm{I}, \mathrm{O}_{2}$ achieved was checked by breath to breath measurement over $20 \mathrm{~min}$ in 10 normal volunteers.

\section{Protocol}

The protocol used to assess flight fitness and the algorithm used in the interpretation of the results are summarized in figure 1. Patients remained seated throughout the assessment. During a $10 \mathrm{~min}$ acclimatization period the purpose of the assessment was explained to patients and an ear probe attached to a pulse oximeter (Model 3740; Ohmeda, Guildford, UK). Patients were then fitted with a full face $35 \%$ Venturi mask to enable hypoxic challenge to be performed. Under this mask, nasal cannulae were also fitted, which were only used (when required by the algorithm) to answer the secondary question "if simulated altitude results in hypoxaemia could this be corrected by the administration of supplemental oxygen during flight?"

Baseline arterial oxygen salvation $\left(\mathrm{Sa}_{2} \mathrm{O}_{2}\right)$ was recorded every $30 \mathrm{~s}$ for 5 min whilst breathing room air. If $\mathrm{Sa}_{\mathrm{a}} \mathrm{O}_{2}$ was $<90 \%$ during the baseline period then no HIT was performed and the patients were informed that they were at risk of experiencing respiratory distress during flight and advised against flying (group $\mathrm{C}$ ).

If baseline $\mathrm{Sa}_{\mathrm{a}} \mathrm{O}_{2}$ was $\geq 90 \%$ an HIT was performed by the patient breathing for $20 \mathrm{~min}$ through a 35\% Venturi mask supplied with $8 \mathrm{~L} \cdot \mathrm{min}^{-1}$ of $100 \%$ nitrogen from a cylinder (BOC, Guilford, UK). If $\mathrm{Sa}_{2} \mathrm{O}_{2}$ remained $\geq 90 \%$ dur-ing the HIT then the patient was classed as fit to fly (group A). 


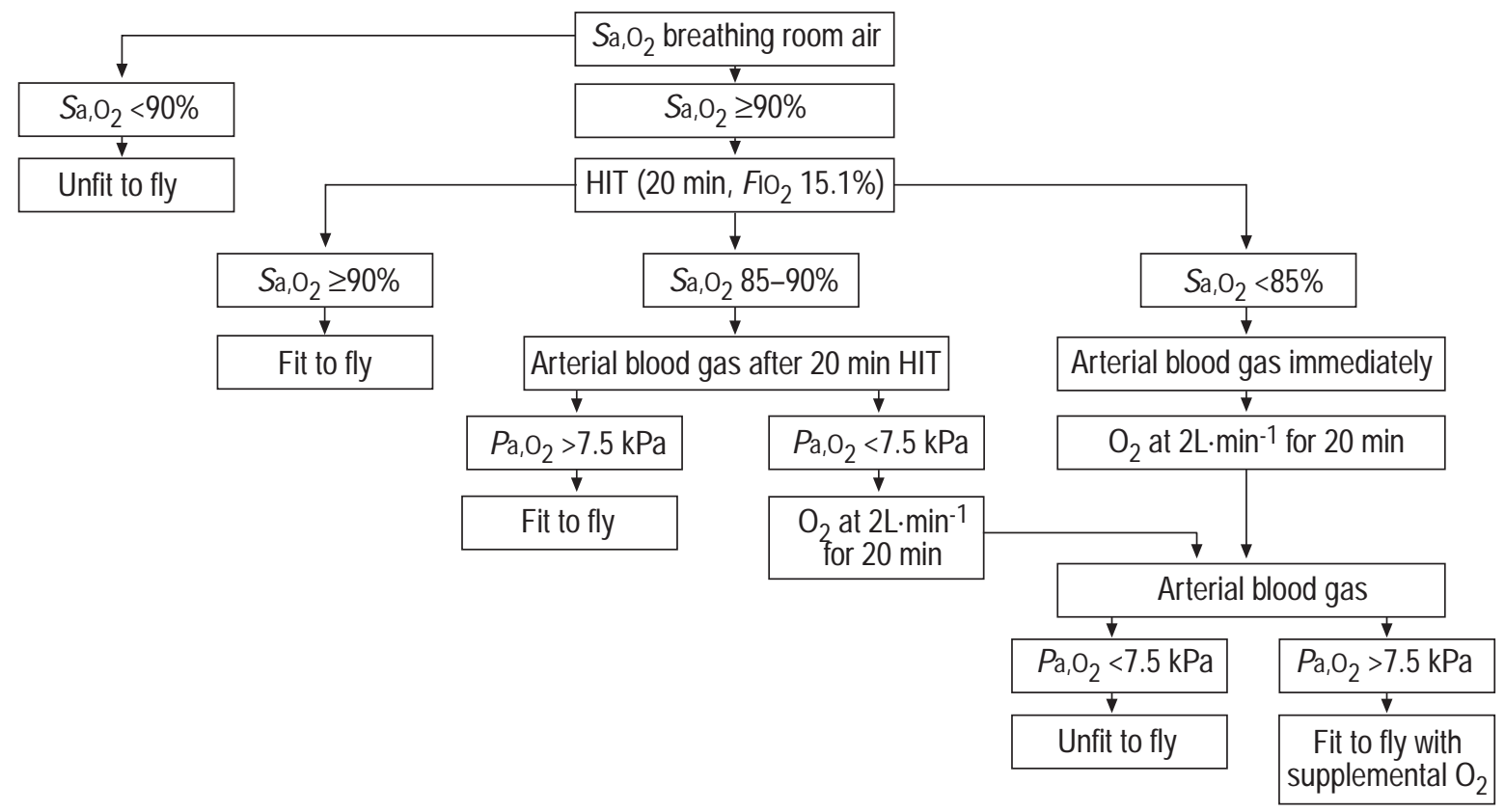

Fig. 1. - Baseline arterial oxygen saturation $\left(\mathrm{Sa}_{\mathrm{a}} \mathrm{O}_{2}\right)$ was measured whilst breathing air for 5 min. HIT: hypoxia ventilation test; $F \mathrm{I}, \mathrm{O}_{2}$ : inspiratory oxygen fraction; $P \mathrm{a}_{2} \mathrm{O}_{2}$ : arterial oxygen pressure; A: fit to fly; $\mathrm{B}$ : fit to fly with supplemental oxygen; C: unfit to fly.

If $\mathrm{Sa}_{\mathrm{a}} \mathrm{O}_{2}$ fell below $90 \%$ during $\mathrm{HIT}$, the degree of hypoxaemia was measured by arterial blood gas (ABG). This was done immediately if $\mathrm{Sa}, \mathrm{O}_{2}$ fell below $85 \%$ or after $20 \mathrm{~min}$ of HIT if the saturation was $85-90 \%$. If arterial oxygen pressure $\left(\mathrm{Pa}_{\mathrm{a}} \mathrm{O}_{2}\right)$ was $>7.5 \mathrm{kPa}$ then the patient was considered "fit to fly" and was placed in group A. If $P \mathrm{a}, \mathrm{O}_{2}$ was $<7.5 \mathrm{kPa}$ then the patient went on to be assessed for fitness to fly with supplemental oxygen. To do this they received supplemental $\mathrm{O}_{2}$ via the nasal cannulae at a flow rate of $2 \mathrm{~L} \cdot \mathrm{min}^{-1}$ for $20 \mathrm{~min}$ whilst still breathing the hypoxic gas mixture through the Venturi mask. At the end of the 20 min period a second $A B G$ was obtained and if $P \mathrm{a}, \mathrm{O}_{2}$ remained $<7.5 \mathrm{kPa}$ then patients were assessed as "unfit to fly" (group C). If $P \mathrm{a}, \mathrm{O}_{2}$ was $>7.5 \mathrm{kPa}$ then patients were assessed as "fit to fly with supplemental oxygen" and were placed in group B.

In an attempt to validate the protocol, a questionnaire was sent to all patients who had undergone assessment for completion upon return from their travel. The questionnaire comprised six questions. They were asked if there had been any change in their clinical condition between the date of the assessment and the date of travel, whether they had experienced any respiratory distress during their flight (either whilst seated or when moving around the plane) and also the duration of the flight. In addition, patients were asked if they had required attention from cabin staff during the flight because of their breathing and whether they had received oxygen. Where patients had been assessed as "unfit to fly" their intentions regarding future air travel were obtained.

\section{Results}

All patients tolerated the assessment well without exacerbation of symptoms. Of the 28 patients who attended the laboratory for assessment, 22 patients $(78 \%)$ were in group A (fit to fly) with a further two (7\%) in group B (fit to fly with supplemental $\mathrm{O}_{2}$ ) and the remaining four patients (14\%) in group C (unfit to fly). All patients in this latter group had pretest ear $\mathrm{O}_{2}$ saturations $<90 \%$.

\section{Validation of hypoxic challenge method}

$\mathrm{FI}_{\mathrm{I}} \mathrm{O}_{2}$ was measured in four volunteers wearing $40 \%$ Venturi masks and was found to be $14.7 \pm 0.3 \%$. When measured in volunteers wearing $35 \%$ Venturi masks, $\mathrm{F} \mathrm{I}_{2} \mathrm{O}_{2}$ was found to be very stable at the desired value $(15.1 \pm$ $0.2 \%$ mean $\pm \mathrm{SD}$ of measurements made every $30 \mathrm{~s}$ in 10 subjects for 20 min per subject). Because of the high flow into the mask, the $F \mathrm{I}, \mathrm{O}_{2}$ did not appear to be influenced by the patients' minute ventilation or respiratory pattern. As a result of this preliminary study $35 \%$ Venturi masks were used in all patients undergoing assessment.

\section{Pulse oximetry}

Typical traces of $\mathrm{Sa}_{\mathrm{a}} \mathrm{O}_{2}$ for patients in groups $\mathrm{A}$ and $\mathrm{B}$ are shown in figure 2. In group A mean baseline $\mathrm{Sa}_{\mathrm{a}} \mathrm{O}_{2}$ was $96.4 \pm 1.0 \%$ at the completion of the HIT mean $\mathrm{Sa}_{\mathrm{a}} \mathrm{O}_{2}$ had fallen to $92.7 \pm 1.7 \%(\mathrm{p}<0.01$, paired t-test). There was no statistically significant difference between baseline $\mathrm{Sa}_{\mathrm{a}} \mathrm{O}_{2}$ in patients from groups A and B $(96.4 \pm 1.0 \%$ and $94.3 \pm$ $1.8 \%$ respectively).

\section{Arterial blood gas analysis}

Both patients with group $\mathrm{B}$ results (patients 7 and 18) had a $P \mathrm{a}, \mathrm{O}_{2}<7.5 \mathrm{kPa}$ at ABG1 (5.29 (patient 7) and 6.01 $\mathrm{kPa}$ (patient 18) respectively), which recovered during the administration of supplemental $\mathrm{O}_{2}$ to reach 9.14 (patient 7) and 9.29 (patient 18) $\mathrm{kPa}$ after $20 \mathrm{~min}$. Baseline values for 
a)

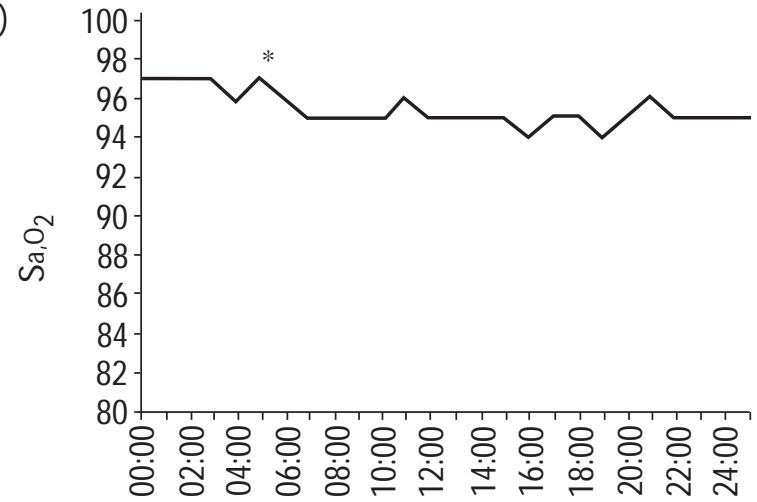

b)

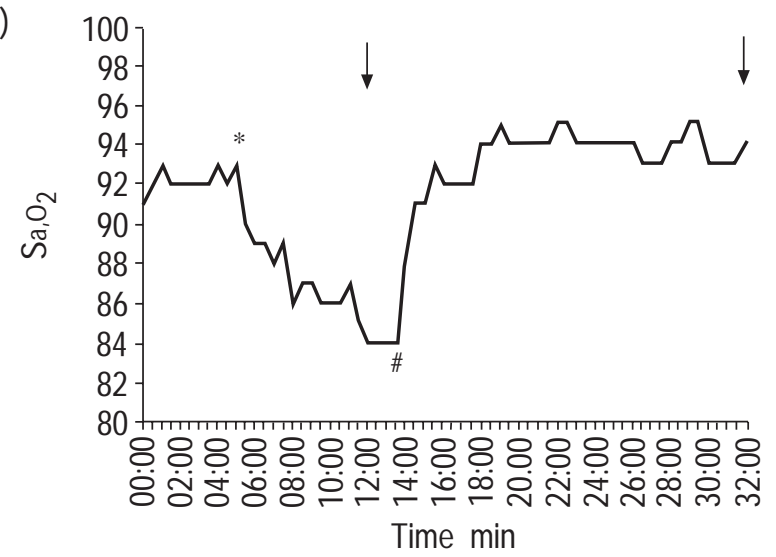

Fig. 2. $-\mathrm{Sa}, \mathrm{O}_{2}$ tracings from a patient in a) group $\mathrm{A}$ and b) group B. *: patient begins to breathe through Venturi mask; ${ }^{\text {\#}}$ : supplemental $\mathrm{O}_{2}$ delivered via nasal cannulae at $2 \mathrm{~L} \cdot \mathrm{min}^{-1} . \downarrow$ : arterial blood gas sample collected.

arterial carbon dioxide pressure $\left(\mathrm{P}_{\mathrm{a}, \mathrm{O}_{2}}\right)$ were 7.67 and 4.56 $\mathrm{kPa}$ for patients 7 and 18 respectively. After supplemental $\mathrm{O}_{2} \mathrm{Sa}_{2} \mathrm{CO}_{2}$ was $6.92 \mathrm{kPa}$ in patient 7 and $4.84 \mathrm{kPa}$ in patient 18.

\section{Questionnaire response}

Twenty-four patients completed and returned a questionnaire. The results are summarized in table 2 . No patients reported any significant change in their clinical condition between the date of the assessment and the date of travel. One patient in group A had a panic attack while on the plane, which was treated with supplemental $\mathrm{O}_{2}$ for $5 \mathrm{~min}$. One patient in group $\mathrm{B}$ flew against medical advice without supplemental $\mathrm{O}_{2}$ and reported no problems during either of their 6-h flights. There was a mean period of $33 \pm 41$ days between the date of the assessment and the date of the first flight (range 6-122 days). Mean flight time was 6 h $10 \mathrm{~min}$ (range 3-16 h).

\section{Discussion}

As commercial air travel continues to expand, more patients with chronic respiratory disease are likely to consider travel by air. Identification of patients who are at risk of developing respiratory distress in the reduced $\mathrm{PO}_{2}$ of a pressurized aircraft might prevent unnecessary discomfort for the patient during the flight either by the provision of supplemental $\mathrm{O}_{2}$ whilst on board or by suggesting the patient should reconsider their travel plans.

It was found that all of the patients who were assessed as unfit for air travel using our protocol accepted the results of the assessment and altered their plans accordingly. The authors are aware of one patient who flew without supplemental $\mathrm{O}_{2}$ despite our recommendation that they should have this facility available, who then reported no significant changes in symptoms during a $6 \mathrm{~h}$ flight (table 2).

BYRNE [12], reporting on the use of supplemental $\mathrm{O}_{2}$ on commercial aircraft found that for every two passengers who had arranged for the provision of supplemental $\mathrm{O}_{2}$ during flight another passenger received $\mathrm{O}_{2}$ for symptomatic relief when it had not been prearranged. The reasons for these requests for unscheduled $\mathrm{O}_{2}$ therapy varied widely and without in-flight saturation measurements it is difficult to determine what proportion of these incidents could have been prevented by preflight HIT assessment.

Are hypoxic challenges necessary to predict a person's flight fitness or can problems be predicted from other measurements such as lung function tests? Individual responses will depend on patients' sensitivity to hypoxaemia and their capacity to increase alveolar ventilation (in the face of airflow obstruction) to compensate for the hypoxia. Both hypoxic sensitivity and the resulting ventilatory response will vary between individuals in a way which will not be predictable from FEV1. A more reliable way to predict in-flight hypoxaemia is to perform some form of hypoxic challenge. Our results support this view. For example, the patient with the lowest FEV1 and \% predicted FEV1 did not desaturate during hypoxia. Although all but one of the patients who did desaturate had FEV1 $<1 \mathrm{~L}$, there were an equal number with FEV $1<1 \mathrm{~L}$ in the group that did not desaturate during HIT. One patient with chronic obstructive pulmonary disease, (COPD) with an FEV1 as high as $1.85 \mathrm{~L}$ did desaturate. The present results also show pairs of patients with the same diagnosis, matching FEV1 and \% predicted FEV1 where one patient desaturated and the

Table 2. - Results of postal questionnaire

\begin{tabular}{|c|c|c|}
\hline $\begin{array}{l}\text { Group A } \\
\text { Fit to fly } \\
n=22\end{array}$ & $\begin{array}{l}\text { Group B } \\
\text { Fit to fly with supplemental } \mathrm{O}_{2} \\
\mathrm{n}=2\end{array}$ & $\begin{array}{l}\text { Group C } \\
\text { Unfit to fly } \\
n=4\end{array}$ \\
\hline $\begin{array}{l}14 \text { patients flew with no problems reported. } \\
1 \text { patient had a panic attack, treated } \\
\text { with supplemental } \mathrm{O}_{2} \text { for } 5 \text { min. } \\
4 \text { patients have not yet flown. } \\
3 \text { patients abandoned travel plans. }\end{array}$ & $\begin{array}{l}1 \text { patient flew without supplemental } \\
\mathrm{O}_{2} \text { with no problems reported. } \\
1 \text { patient abandoned travel plans. }\end{array}$ & All 4 patients abandoned travel plans. \\
\hline
\end{tabular}


other did not (e.g. patients 10 and 11 in table 1). On clinical grounds it was decided that challenging patients with a pretest saturation $<90 \%$ was not appropriate because these patients would be at risk in the event of failure or accidental disconnection of supplemental oxygen in flight. However of those with pretest saturation $\geq 90 \%$ who were challenged, there was no difference in pretest saturation between those who desaturated on challenge and those who did not. Thus in patients who are not desaturated $(<90 \%)$, at rest it is inappropriate to attempt to predict desaturation during flight from measurements made without hypoxic challenge.

Although the authors have not yet formally assessed the reproducibility of the test, two patients (patients 8 and 17 in table 1) have had the test repeated 5 and 12 months, respectively, after initial testing. The results of the second tests were identical to those of the first (one patient in group $\mathrm{A}$, one patient in group $\mathrm{C}$ ) These preliminary findings suggest that the test does appear to be reproducible although more data are needed.

The HIT has been used for a number of years as both a research tool and for clinical assessment. Does reducing the $F \mathrm{I}, \mathrm{O}_{2}$ mimic appropriately the effect of reduced absolute pressure in an aircraft? DiLlard et al. [13] found the HIT produced comparable results to exposure in a hypobaric chamber. Because of the relative inaccessability of hypobaric chambers the development of a simple HIT which can be used in respiratory function laboratories for the preflight assessment of patients is potentially beneficial. To ensure the protocol would be applicable in routine laboratories, the use of a plethysmograph [3] and expensive cylinders of hypoxic gas mixtures was avoided. The simple and elegant method of VoHrA and KLOCKE [11] using a Venturi mask supplied with nitrogen was therefore adopted. The authors suggested that a $40 \%$ Venturi mask should be used to ensure that the $\mathrm{FI}, \mathrm{O}_{2}$ was maintained at $15.1 \%$ during the HIT. During preliminary testing $F \mathrm{I}, \mathrm{O}_{2}$ was sampled at the mouth, while patients breathed on the mask and found that the particular brand of $35 \%$ mask the authors used could generate a consistent $F \mathrm{I}_{\mathrm{O}_{2}}$ of $15.1 \%$, but this should be checked for a given brand of mask before use.

In the protocol described in this paper the authors chose not to perform arterial blood gas analysis in all patients to improve the patient acceptability of the assessment. By only using blood gas analysis in patients when $\mathrm{Sa}_{\mathrm{a}} \mathrm{O}_{2}$ fell $<90 \%$ during the HIT they reduced the number of ABG needed by $93 \%$.

The definition and validation of an appropriate algorithm to guide patients from the results of a hypoxic challenge is notoriously difficult. It is not clear what resting $\mathrm{PO}_{2}$ constitutes a hazard or a high probability that symptoms may develop, and this is likely to vary between individuals. The algorithm employed in the present study is deliberately conservative to reduce the chance of unpredicted respiratory problems during flight. Prospective validation of the algorithm by measurement of symptoms and oxygenation during flight would be hazardous and costly, and laboratory simulation of prolonged flight would also be a major undertaking. The present algorithm makes several assumptions which have not yet been tested. In particular, it is assumed that a short exposure to hypoxia in the laboratory can predict problems occurring in a flight lasting several hours. A further concern is that patients who are well at rest may become unwell when mobilizing at altitude (e.g. to use the aircraft toilet), particularly if they are depending on supplemental oxygen which is disconnected when they mobilize. Finally the results of the assessment may be misleading if the patient's condition changes between the day of the assessment and the day of the flight. All these factors need to be borne in mind when advising patients about the results of an HIT. Follow-up questionnaires may be helpful in providing some validation of the protocol, but will not show how many of those advised not to fly might have been able to travel successfully.

Most published studies have only studied patients with COPD and have excluded hypercapnic patients although there is no specific exclusion in published COPD care guidelines $[14,15]$. Although the majority of the patients in this study did have a diagnosis of COPD assessed patients with other respiratory diseases were also assessed. Both patients in group B were diagnosed with COPD and arterial sampling showed mild hypercapnia in one patient (patient 7 in table 1). Neither patient supplied with supplemental oxygen developed significant additional hypercapnia. Further research into the ventilatory response of hypercapnic patients at altitude is required.

In summary, the use of a hypoxia inhalation test on a range of patients with chronic respiratory disease, within a clinical respiratory function laboratory environment, is reported. A locally derived algorithm has been used for the interpretation of the assessment and found that although the majority of patients should be able to travel safely without supplemental $\mathrm{O}_{2}$, others become significantly hypoxic during challenge and, importantly, it is not possible to predict a hypoxic response from measurements of spirometry or pretest saturation.

\section{References}

1. Department of the Environment, Transport and the Regions website: www.aviation.detr.gov.uk/aed/air/air3.

2. Cottrell JJ. Altitude exposures during aircraft flight. Flying higher. Chest 1988; 92: 81-84.

3. Cramer D, Ward S, Geddes D. Assessment of oxygen supplementation during air travel. Thorax 1996; 51:202203.

4. Gong H, Tashkin DP, Lee EY, Simmons MS. Hypoxiaaltitude simulation test. Evaluation of patients with chronic airway obstruction. Am Rev Respir Dis 1984; 130: 980 986.

5. Oades PJ, Buchdahl RM, Bush A. Prediction of hypoxaemia at high altitude in children with cystic fibrosis. BMJ 1994; 308: 15-18.

6. Berg BW, Dillard TA, Derderian SS, Rajagopal KR. Hemodynamic effects of altitude exposure and oxygen administration in chronic obstructive pulmonary disease. JAMA 1993; 94: 407-412.

7. Dillard TA, Rajagopal KR, Slivka WA, Berg BW, Mehm WJ, Lawless NP. Lung function during moderate hypobaric hypoxia in normal subjects and in patients with chronic obstructive pulmonary disease. Aviat Space Environ Med 1998; 69: 979-985.

8. Dillard TA, Berg BW, Rajagopal KR, Dooley JW, Mehm WJ. Hypoxaemia during air travel in patients with chronic obstructive pulmonary disease. Ann Intern Med 1989; 111: 362-367. 
9. Crapo RO, Morris AH, Gardner RM. Reference spirometric values using techniques and equipment that meet ATS recommendations. Am Rev Respir Dis 1981; 123: 659-664.

10. Hall AM, Heywood C, Cotes JE. Lung function in healthy British women. Thorax 1979; 34: 359-365.

11. Vohra KP, Klocke RA. Detection and correction of hypoxaemia associated with air travel. Am Rev Respir Dis 1993; 148: 1215-1218.

12. Byrne NJ. Comparison of airline passenger oxygen systems. Aviat Space Environ Med 1995; 66: 780-783.
13. Dillard TA, Moores LK, Bilello KL, Phillips YY. The preflight evaluation. A comparison of the hypoxia inhalation test with hypobaric exposure. Chest 1995; 107: 352-357.

14. Bouros D, Tzanakis N. Evaluation of chronic obstructive pulmonary disease patients for air travel and surgery. Eur Respir Mon 1998; 7: 278-287.

15. American Thoracic Society. Standards for the diagnosis and care of patients with chronic obstructive pulmonary disease. Am J Respir Crit Care Med 1995; 152 (Suppl. 5): S113. 